\title{
How can smart technologies contribute to sustainable product lifecycle management?
}

Yang Liu, Yingfeng Zhang, Shan Ren, Miying Yang, Yutao Wang and Donald Huisingh

The self-archived postprint version of this journal article is available at Linköping University Institutional Repository (DiVA):

http://urn.kb.se/resolve?urn=urn:nbn:se:liu:diva-163444

N.B.: When citing this work, cite the original publication.

Liu, Y., Zhang, Y., Ren, S., Yang, M., Wang, Y., Huisingh, D., (2020), How can smart technologies contribute to sustainable product lifecycle management?, Journal of Cleaner Production, 249, 119423. https://doi.org/10.1016/j.jclepro.2019.119423

Original publication available at:

https://doi.org/10.1016/j.jclepro.2019.119423

Copyright: Elsevier

http://www.elsevier.com/

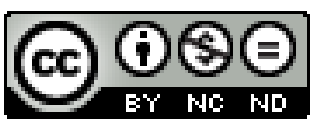




\section{Journal Pre-proof}

How can smart technologies contribute to sustainable product lifecycle management?

Yang Liu, Yingfeng Zhang, Shan Ren, Miying Yang, Yutao Wang, Donald Huisingh

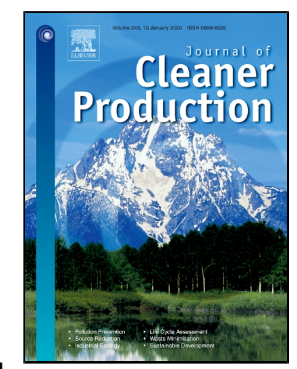

PII:

S0959-6526(19)34293-3

DOI:

https://doi.org/10.1016/j.jclepro.2019.119423

Reference:

JCLP 119423

To appear in:

Journal of Cleaner Production

Received Date:

20 November 2019

Accepted Date:

22 November 2019

Please cite this article as: Yang Liu, Yingfeng Zhang, Shan Ren, Miying Yang, Yutao Wang, Donald Huisingh, How can smart technologies contribute to sustainable product lifecycle management?, Journal of Cleaner Production (2019), https://doi.org/10.1016/j.jclepro.2019.119423

This is a PDF file of an article that has undergone enhancements after acceptance, such as the addition of a cover page and metadata, and formatting for readability, but it is not yet the definitive version of record. This version will undergo additional copyediting, typesetting and review before it is published in its final form, but we are providing this version to give early visibility of the article. Please note that, during the production process, errors may be discovered which could affect the content, and all legal disclaimers that apply to the journal pertain.

(C) 2019 Published by Elsevier. 


\section{How can smart technologies contribute to sustainable product lifecycle management?}

Yang Liu ${ }^{\mathrm{a}, *}$, Yingfeng Zhang ${ }^{\mathrm{b}, *}$, Shan Ren ${ }^{\mathrm{b}}$, Miying Yang ${ }^{\mathrm{c}}$, Yutao Wang ${ }^{\mathrm{d}}$, Donald Huisingh ${ }^{\mathrm{e}}$

${ }^{a}$ Department of Management and Engineering, Linköping University, Sweden

${ }^{\mathrm{b}}$ Key Laboratory of Contemporary Design and Integrated Manufacturing Technology, Ministry of Education, Northwestern Polytechnical University, China

${ }^{c}$ College of Engineering, Mathematics and Physical Sciences, University of Exeter, UK

${ }^{\mathrm{d}}$ Department of Environmental Science and Engineering, Fudan University, China

e Institute for a Secure and Sustainable Environment, University of Tennessee, USA

* Corresponding Author: yang.liu@liu.se (Y. Liu), zhangyf@nwpu.edu.cn (Y. Zhang)

\section{Abstract}

This Virtual Special Issue (VSI) was proposed on par with the fascinating and exponentially growing development of smart enabling technologies, such as Internet of Things (IoT), Cyber-Physical System (CPS), Cloud Computing (CC), Artificial Intelligence (AI), Big Data Analytics (BDA), Digital Twin (DT), etc, which have greatly advanced the development of sustainable smart manufacturing throughout the lifecycle. The VSI addressed issues that were not properly or even incorrectly addressed in the existing literature. The authors of this VSI sought to introduce new knowledge and debates to lead the research directions to new paths. The editorial team invited well-established researchers in this area and received about 40 highly qualified submissions, out of which 12 were accepted after standard peer-review procedure of the Journal of Cleaner Production, which covered the three main themes defined in the "Call-for-Papers". The contributing authors were from Brazil, China, Finland, Pakistan, Sweden, USA (in alphabetical order). The coordinators of this VSI are confident that the contents of this VSI will advance the science of digitalisation and will help society to make real progress towards sustainable societies.

Keywords: sustainable product lifecycle management; smart technologies; sustainable smart manufacturing; editorial

\section{Introduction}

Research on smart enabling technologies is attracting the attention of more and more researchers. The new approaches are increasingly studied and applied across a wide variety of industries. These new approaches are contributing to the future of cleaner production (CP) as well. Many believe or hope these technologies will change the future for good. Most of the previous studies were focussed upon utilizing smart technologies to improve the planning, design, operations and maintenance of production systems as well as on improving products/services for $\mathrm{CP}$, and the coordinators of this VSI are confident that this trend will continue. However, there are some issues that are not properly addressed in the existing literature. For example, firstly, many existing studies focused only on applying smart enabling technologies to facilitate smart manufacturing, but the sustainability of the whole product lifecycle management (PLM) processes was seldom addressed. Secondly, for existing special issues published in other journals, studies on integrated applications of various smart enabling technologies to enhance the sustainability of PLM was nearly absent. Thirdly, few researchers performed investigations covering smart enabling technologies in the whole lifecycle (such work was especially lacking in the design and recovery stages). These issues led to the following research questions:

- What are the important elements and enablers for sustainable smart manufacturing and sustainable PLM?

- How to effectively utilize smart enabling technologies to realize the sharing and integration of data and manufacturing in different lifecycle stages, and to facilitate sustainable PLM?

- What benefits can be brought to industrial communities by penetrating and using smart enabling technologies into the whole lifecycle?

The authors of the papers in this VSI addressed these issues and research questions, with state-of-the-art theories, methods, and technologies, to effectively integrate and apply smart enabling technologies for using lifecycle big data to promote the implementation of sustainable production and PLM.

With the increasing availability of these emerging technologies, massive data are available in different lifecycle phases with various degrees of complexity, thereby providing a solid foundation for further improvements (S. Ren et al., 2019). The volume of data for product lifecycle has increased significantly with increasing applications of smart technologies (Lou et al., 2018). The benefits of lifecycle big data have spread throughout the entire product value chain thereby, e.g. improving the product-service design, enhancing production performance at the shop floor, and by enhancing the capacity for predictive maintenance etc. (Zhang et al., 2017a). 
The editorial team anticipates great potential for the usage of these technologies for improving CP implementation in companies throughout the lifecycle and for achieving sustainability from a system's perspective. For example, IoT can collect real-time data from existing scenarios, and together with BDA and AI can extract and analyse useful information from e.g. market survey, financial figures, lifecycle data to identify customer behaviour and understand the value for stakeholders and how to provide the values, and better reveal the relevant market segment and obligatory properties in terms of timeliness, accuracy, and broadness. The real-time customer feedback and market survey will enhance the fulfilment of requirements and value creation. The market analyses can reveal more details of the value chain of stakeholders to better describe the requirements and to identify hidden criteria. Studies such as (Gupta et al., 2018) utilized BDA as a fundamental basis for informed and data-driven decision making in supporting CE from a stakeholder perspective. Digital twins, based virtual models can boost smart manufacturing from design to operations (Tao and Qi, 2019).

These technologies can help company managers to make improvements in the key process areas, such as remote machine setup; corrective and predictive maintenance; material supply; product pricing; and information reporting (Zancul et al., 2016). Such improvements are valuable for optimizing and balancing the trade-offs between product performance vs. service aspects such as more accurate prediction on maintenance, optimizing the service schedules, and the trade-offs between product performance vs. environmental performance. The optimal solutions obtained can be further evaluated based on BDA and AI in addition to traditional evaluation methods. For instance, data analytics can provide insights so that more objective and accurate decisions can be made. However, we must be careful to also address the potential negative effects and work to minimize them.

\section{Themes in this VSI}

Articles in this VSI are focussed upon diverse product lifecycle phases such as product design, production, service and maintenance, while we also welcome submissions that cover other lifecycle phases.

\subsection{Design}

One paper clearly fit into this theme.

The article "Turning part design for joint optimisation of machining and transportation energy consumption" by $\mathrm{Hu}$ et al., (2019) addressed the design phase to optimize energy consumption. In manufacturing processes, machining energy consumption (MEC) and transportation energy consumption (TEC) are two major sources of energy use. They are highly affected by the design parameters of the parts. The authors investigated, in the product design phase, ways to optimize energy consumption in machining and transportation. A TEC model was developed and embedded into a modified MEC, to obtain a machine-vehicle system energy consumption (MVSEC) model. Then they used an integrated optimal design of diameter and cutting parameters to minimize the reduction of MEC and TEC. They tested the model in an experiment of a shaft sleeve design, the simulation of which showed that using the proposed optimization method can achieve a 3.53\% MVSEC reduction. This provides designers insights into ways the design parameters are important for obtaining optimized energy consumption solutions.

\subsection{Production/manufacturing}

A large portion of existing research has been focussed on this phase and that was reflected in the inputs for this: six papers formed the biggest theme of this VSI.

The article "An 'Internet of Things' enabled dynamic optimization method for smart vehicles and logistics tasks" by Liu et al., (2018) applied smart technologies for sustainable logistics. As pointed by Chhabra et al., (2018), green logistics is an important trend of development to reduce environmental risks of logistical operations and thereby establish a balance among economic, environmental and social objectives. Currently, the typical challenge that the logistics industry faces is the lack of real-time information on logistics resources and the sharing of information (Ding et al., 2018; Muñoz-Villamizar et al., 2018). This has resulted in increased logistics costs, and low-efficiency allocation of logistical resources (Qian et al., 2020; J. Wang et al., 2019). To deal with these challenges, the authors applied IoT technology to the logistics delivery process to achieve dynamic optimization of logistics tasks. An IoT-enabled real-time status sensing model of logistics vehicles was developed and it enabled vehicle managers to collect real-time information on logistics resources. Then a dynamic optimization method for smart vehicles and logistical tasks was developed to improve the utilization of logistical resources and to realize a better balance among the three dimensions of sustainability. 
The article "Optimization of cutting parameters considering tool wear conditions in low-carbon manufacturing environment" by Tian et al., (2019) studied a classic case in cleaner production that needs to balance the material waste and carbon emission. By introducing tool wear conditions as an additional decision variable, the authors developed a quantitative, multi-objective, low carbon-oriented, cutting parameters optimization method considering different tool wear conditions based on the production carbon emissions, cost and time so that the proposed method accorded more with the practical circumstances and reduced more carbon emissions globally. The method used for this optimization was the "Non-dominated Sorting Genetic Algorithm II (NSGA-II)," combined with game theory to improve the Pareto solutions and to overcome the limits of traditional optimization methods. To demonstrate the feasibility and advantages, they conducted a case study of the proposed method on real manufacturing industrial data and compared it with other optimization methods. From that, they summarized three observations. The manufacturing companies can easily and conveniently apply the proposed method to achieve reduced costs increased product competitiveness, and reduced trade barriers.

The article "Towards energy and material efficient laser cladding process: Modeling and optimization using a hybrid TS-GEP algorithm and the NSGA-II" by Peng et al., (2019) dealt with additive manufacturing which is an important digital manufacturing technology applied in many high-tech industries. The authors studied the material and energy efficiency issue in the additive manufacturing process. They developed an integrated Tabu search and gene expression programming (TS-GEP) algorithm for predictive modelling of energy consumption and metallic powder usage rate in laser cladding process of additive manufacturing. Based on the model, they used nondominated sorting genetic algorithm II (NSGA-II) to provide optimization which can assist in parameter selection of laser cladding processes. The model was tested in the laser cladding process, and the optimized set of processing parameters for energy and material utilisation was developed. The novelty is the development of TS-GEP and NSGA-II algorithms, and the results can be used to facilitate parameter selection for optimized material and energy efficiency in the additive manufacturing process.

The article "An optimization model for energy-efficient machining for sustainable production" by Wang et al., (2019) addressed the optimization of the machining process and its contribution to sustainable production. Currently, a more environmentally-friendly production and management mode is essential to reduce energy consumption and environmental impact of the manufacturing industry (Zhang et al., 2017b). Therefore, research on sustainable product lifecycle management (SPLM), especially, on sustainable production has been increasingly focused upon recently. Energy-efficient machining through optimizing machining schemes is an important approach for a more sustainable production in manufacturing enterprises. However, how to identify the best combination of machining schemes is a challenge. In addition, a comprehensive energy optimization approach for manufacturing parts is deficient. To address these challenges, the authors selected the Standard for the Exchange of Product model data-Numerical Control (STEP-NC) as the enabling technology to calculate the energy consumption and to obtain the machining energy of a whole part from a holistic perspective. Then a model was established to optimize the energy consumption by adjusting machining resources, parameters, strategies, operations, etc. An improved ant colony optimization (ACO) algorithm, which consists of encoding and decoding, initialization, machining scheme generation, evaluation and update, was developed to solve the model effectively.

The article "Makerchain: A blockchain with chemical signature for self-organizing process in social manufacturing" by Leng et al., (2019) utilised blockchain in the context of social manufacturing. Various distributed makers are involved in the product design, manufacturing and assemblies (Hamalainen et al., 2018). This makes the trust among manufacturers a challenge. Blockchain technology has the potential to help to ensure trust and secured collaboration among the decentralised, autonomous manufacturers. In this study, the authors developed a new block-chain driven Makerchain model, including manufacturing and networking sections, for decentralised mass personalisation. This model can be used to solve the cyber-credit issue of social manufacturing among multiple makers. In this model, they proposed to use chemical signature and consensus algorithm for anti-counterfeiting, to represent unique features of personalized products. The signature data are then twinned to blockchain and databases, which can increase the trustworthiness of the transactions among decentralised makers. Then a smart contract tree for the self-organizing process in Makerchain was developed to enable the automatic transactions among makers and the third-party verification of product lifecycles. Finally, they developed a Makerchain Decentralized Application to demonstrate that through this method the decentralised makers can self-organize themselves to meet personalized demands.

The article "An emergy-based sustainability evaluation method for outsourcing machining resources" by Cai et al., (2019) dealt with production planning, which is after design but before production. The authors investigated 
the sustainability evaluation method for outsourcing machining resources for the purpose of improving resource efficiency. They reviewed the existing studies on outsourcing machining resources and proposed an emergy-based sustainability evaluation method for outsourcing planning. The method includes four emergy models, i.e. production quality emergy, production time emergy, production logistics cost emergy and production resources consumption emergy. The optimal outsourcing machining plan can be generated based on an integrated assessment of the four emergy models. This method was tested and the results can be used for sustainability evaluation and selection of the outsourcing machining resources, in practice.

\subsection{Service, maintenance and other lifecycle phases}

This theme covers five papers dealing with lifecycle phases other than the above themes.

The review article "A comprehensive review of big data analytics throughout product lifecycle to support sustainable smart manufacturing: A framework, challenges and future research directions" by S. Ren et al., (2019) provided a general overview of the motivations and challenges of this VSI. With the rapid development of advanced information and communication technology (ICT), smart manufacturing (SM) has received increased attention from academia and industry. Emerging technologies provide the opportunity for manufacturing companies to integrate their products and services to implement sustainable production strategies (Tukker, 2015). As one of the most important technologies for SM, BDA can help industrial leaders to make more-informed business decisions. However, by analysing the existing literature, SM and BDA were individually researched, and the sustainability and SM were also investigated separately. To overcome these limitations, a comprehensive review was conducted by the authors to develop a theoretical foundation and to further provide scientific insights in this area. The term sustainable smart manufacturing (SSM) was first coined and defined. The key enabling technologies of SM, and especially, the applications of BDA in SM were reviewed and evaluated. A framework of BDA in SSM was proposed, meanwhile, the advantages of BDA in the whole PLM (i.e. design, manufacturing, maintenance, recovery) were discussed. The findings can help industrial leaders and policy-makers make more ecologically and economically smarter decisions for the whole lifecycle management.

The article "Optimization of energy consumption in coating removal for recycling scrap coated cemented carbide tools using hybrid laser-waterjet" by Yun et al., (2019) dealt with recovery. Considering the importance of energy conservation to contribute to sustainable development, the authors proposed a multi-objective optimization model for hybrid laser-waterjet machines in coating removal to increase energy efficiency and productiveness. Specifically, the cutting specific energy consumption (CSEC) and the processing time were balanced by transforming the model into a single-objective model and solving it through genetic algorithm. To create the model, the energy consumption models of hybrid laser-waterjet machines were evaluated and the parametric influences were identified by orthogonal experiments, which were used to validate the feasibility of the optimization model through the comparison between single-objective and multi-objective optimization. To document the advantages of the proposed model in environment protection and mechanical properties, they compared it with hightemperature oxidation technologies. Their results showed that (i) the proposed model had a better performance than high-temperature oxidation technologies in energy conservation and substrate quality after processing, (ii) larger feed speed and laser offset distance combined with smaller waterjet distance and pressure could conserve energy, and the model resulted in $80 \%$ energy conservation in minimising CSEC as well as $40 \%$ in minimising processing time. It successfully filled the research gap of energy conservation in laser processing and helped to achieve decoating in a more environmentally friendly way.

The article "Cost-imbalanced hyper parameter learning framework for quality classification" by Zhang et al., (2020) dealt with quality control. The authors proposed an integrated hyper learning framework, which formed an intelligent data-driven quality classification platform, to minimize the average economic losses caused by misclassification; they achieved improved cost-effectiveness in quality control. They addressed the limitations of existing weight classifier training, which was not specific to different types of misclassification and ignored the complex mechanisms between the economic loss and corresponding penalty weight. To solve this problem, they created a framework with two nested layers that allowed various optimization algorithms for hyperparameter identification and different machine learning models with objectives representing diverse concerns or interests. Particle swarm optimization used at the out-layer framework if the differentiability and convexity of the prerequisites were unknown. To test this framework, case studies based on five datasets from different manufacturing and service industries were performed by comparing the economic losses and additional carbon emissions with the other three models considering four different settings of penalty costs due to misclassification. 
Their results indicated that the proposed framework could deliver enhanced robustness on various costs, and was superior to the other three models in costs and in most aspects of environmentally-related performance.

The article "An efficient metaheuristics for a sequence-dependent disassembly planning" by Y. Ren et al., (2019) dealt with remanufacturing and recovery. The authors proposed a disassembly method used AND/OR graph (AOG) which was based on an improved genetic algorithm (IGA) to solve a sequence-dependent disassembly planning problem to improve the economic benefits in more sustainable manufacturing. Firstly, they defined the precedence matrix and the exclusive OR matrix to describe the complex relationships in an AOG and developed a mathematical model to maximize the recovery profit of 'end-of-life,' (EOL) products using an AOG subject to sequence-dependent costs. Then, a two-phase heuristic approach was developed, which consists of eliminating the exclusive OR operations and guaranteeing the precedence constraint, to quickly generate feasible disassembly sequences. To test that the metaheuristic was more efficient in computation time, they compared the CPU time of IGA with two exact algorithms on a series of disassembly problem examples and applied their method to a triaxial five-speed mechanical transmission case. The result showed a $7 \%$ increase in recovery profit and an $11 \%$ decrease in disassembly time. For future studies, they suggested that the uncertainty of the quality of EOL products should be considered in the model so that the robustness of their solutions can be tested.

The article "Big data driven Hierarchical Digital Twin Predictive Remanufacturing paradigm: Architecture, control mechanism, application scenario and benefits" by Y. Wang et al., (2019) also focused on remanufacturing. The authors addressed problems and causes, such as high uncertainty, lack of process tracking management, and focusing mostly on single lifecycle management of remanufacturing paradigm, which is frequently used nowadays. To optimize the remanufacturing process, the new model combined hierarchical digital twin technology and the CPS network to effectively lower the uncertainty in the process. They also implemented smart embedded sensors and devices as tracking perception into the product's multi-lifecycle remanufacturing process to track the multilifecycle process. Then, with the data from tracking perception of the remanufacturing process in multi-lifecycle management, they conducted big data analytics to predict market demand in order to achieve optimization in the configuration of sustainable products and remanufacturing processes. In the end, a case study was conducted based on the automated guided vehicle scenario, and its result validated the feasibility and effectiveness of the proposed new paradigm.

\section{Future prospects}

In summarising the contributions in this VSI, we outlined the future potentials and challenges in the following areas.

1. Data and knowledge based intelligent decision-making throughout the product lifecycle

Under the promotion of the smart enabling technologies, the data and knowledge share among various lifecycle management sectors can be effectively facilitated, and more reasonable and precise decision-making of lifecycle management can be achieved. In an effort to increase the awareness and highlight the areas in which lifecycle big data can provide the cornerstone of industries, future research on data and knowledge based intelligent decision-making throughout the product lifecycle should be conducted but not limited to:

- Data and knowledge based product design, production, operation and maintenance;

- Data and knowledge based supply chain management;

- Data and knowledge based intelligent production decision support systems;

- Data and knowledge based smart manufacturing;

- New concepts, models and methods for sustainable product lifecycle management;

- AI-based manufacturing and operation decision-making;

- Innovative strategies and modes for design, manufacturing, service and maintenance.

2. Digital twin driven, smart product for lifecycle management

Digital twin, as a promising approach to achieve smart manufacturing, is gaining increasing attention. A digital twin refers to a virtual representation of a physical asset or system throughout its lifecycle. The adoption of digital twin technology is becoming more prevalent in the manufacturing industry, including product design, maintenance, and production planning. In the industrial sector, digital twin could foster innovations in product lifecycle management. Future research should include but not be limited to:

- Digital twin in product design, production, operation and maintenance;

- Digital twin in human-machine collaboration;

- Digital twin in sustainable smart manufacturing; 
- Digital twin in product lifecycle data management;

- Digital twin in sustainable product lifecycle management;

- Smart interconnection and interoperation for digital twin;

- Innovative modelling and simulation technologies of digital twin.

We hope this VSI brings insights to open new paths towards digitalisation for the future of cleaner production.

\section{References}

Cai, W., Liu, C., Jia, S., Chan, F.T.S., Ma, M., Ma, X., 2019. An emergy-based sustainability evaluation method for outsourcing machining resources. J. Clean. Prod. 118849. https://doi.org/10.1016/J.JCLEPRO.2019.118849

Chhabra, D., Garg, S.K., Singh, R.K., 2018. Analyzing alternatives for green logistics in an Indian automotive organization: A case study. J. Clean. Prod. 167, 962-969. https://doi.org/10.1016/j.jclepro.2017.02.158

Ding, K., Jiang, P., Su, S., 2018. RFID-enabled social manufacturing system for inter-enterprise monitoring and dispatching of integrated production and transportation tasks. Robot. Comput. Integr. Manuf. 49, 120-133. https://doi.org/10.1016/j.rcim.2017.06.009

Gupta, S., Chen, H., Hazen, B.T., Kaur, S., Santibañez Gonzalez, E.D.R., 2018. Circular economy and big data analytics: A stakeholder perspective. Technol. Forecast. Soc. Change. https://doi.org/10.1016/J.TECHFORE.2018.06.030

Hamalainen, M., Mohajeri, B., Nyberg, T., 2018. Removing barriers to sustainability research on personal fabrication and social manufacturing. J. Clean. Prod. 180, 666-681. https://doi.org/10.1016/J.JCLEPRO.2018.01.099

Hu, L., Liu, W., Xu, K., Peng, T., Yang, H., Tang, R., 2019. Turning part design for joint optimisation of machining and transportation energy consumption. J. Clean. Prod. 232, 67-78. https://doi.org/10.1016/J.JCLEPRO.2019.05.297

Leng, J., Jiang, P., Xu, K., Liu, Q., Zhao, J.L., Bian, Y., Shi, R., 2019. Makerchain: A blockchain with chemical signature for self-organizing process in social manufacturing. J. Clean. Prod. 234, 767-778. https://doi.org/10.1016/J.JCLEPRO.2019.06.265

Liu, S., Zhang, Y., Liu, Y., Wang, L., Wang, X.V., 2019. An 'Internet of Things' enabled dynamic optimization method for smart vehicles and logistics tasks. J. Clean. Prod. 215, 806-820. https://doi.org/10.1016/J.JCLEPRO.2018.12.254

Lou, S., Feng, Y., Zheng, H., Gao, Y., Tan, J., 2018. Data-driven customer requirements discernment in the product lifecycle management via intuitionistic fuzzy sets and electroencephalogram. J. Intell. Manuf. 116. https://doi.org/10.1007/s10845-018-1395-x

Muñoz-Villamizar, A., Santos, J., Montoya-Torres, J.R., Jaca, C., 2018. Using OEE to evaluate the effectiveness of urban freight transportation systems: A case study. Int. J. Prod. Econ. 197, 232-242. https://doi.org/10.1016/j.ijpe.2018.01.011

Peng, S., Li, T., Zhao, J., Lv, S., Tan, G.Z., Dong, M., Zhang, H., 2019. Towards energy and material efficient laser cladding process: Modeling and optimization using a hybrid TS-GEP algorithm and the NSGA-II. J. Clean. Prod. 227, 58-69. https://doi.org/10.1016/J.JCLEPRO.2019.04.187

Qian, C., Zhang, Y., Jiang, C., Pan, S., Rong, Y., 2020. A real-time data-driven collaborative mechanism in fixed-position assembly systems for smart manufacturing. Robot. Comput. Integr. Manuf. 61, 101841. https://doi.org/10.1016/j.rcim.2019.101841

Ren, S., Zhang, Y., Liu, Y., Sakao, T., Huisingh, D., Almeida, C.M.V.B., 2019. A comprehensive review of big data analytics throughout product lifecycle to support sustainable smart manufacturing: A framework, challenges and future research directions. J. Clean. Prod. 210, 1343-1365. https://doi.org/10.1016/J.JCLEPRO.2018.11.025

Ren, Y., Meng, L., Zhang, C., Zhao, F., Saif, U., Huang, A., Mendis, G.P., Sutherland, J.W., 2019. An efficient metaheuristics for a sequence-dependent disassembly planning. J. Clean. Prod. 118644. https://doi.org/10.1016/J.JCLEPRO.2019.118644 
Tao, F., Qi, Q., 2019. Make more digital twins. Nature 573, 490-491. https://doi.org/10.1038/d41586-01902849-1

Tian, C., Zhou, G., Zhang, J., Zhang, C., 2019. Optimization of cutting parameters considering tool wear conditions in low-carbon manufacturing environment. J. Clean. Prod. 226, 706-719. https://doi.org/10.1016/J.JCLEPRO.2019.04.113

Tukker, A., 2015. Product services for a resource-efficient and circular economy - A review. J. Clean. Prod. https://doi.org/10.1016/j.jclepro.2013.11.049

Wang, H., Zhong, R.Y., Liu, G., Mu, W.L., Tian, X., Leng, D., 2019. An optimization model for energy-efficient machining for sustainable production. J. Clean. Prod. 232, 1121-1133. https://doi.org/10.1016/j.jclepro.2019.05.271

Wang, J., Zhang, Y., Liu, Y., Wu, N., 2019. Multi-agent and Bargaining-game-based Real-time Scheduling for Internet of Things-enabled Flexible Job Shop. IEEE Internet Things J. 6, 2518-2531. https://doi.org/10.1109/JIOT.2018.2871346

Wang, Y., Wang, S., Yang, B., Zhu, L., Liu, F., 2019. Big data driven Hierarchical Digital Twin Predictive Remanufacturing paradigm: Architecture, control mechanism, application scenario and benefits. J. Clean. Prod. 119299. https://doi.org/10.1016/J.JCLEPRO.2019.119299

Yun, H., Zou, B., Wang, J., Huang, C., Li, S., 2019. Optimization of energy consumption in coating removal for recycling scrap coated cemented carbide tools using hybrid laser-waterjet. J. Clean. Prod. 229, 104-114. https://doi.org/10.1016/J.JCLEPRO.2019.04.315

Zancul, E. de S., Takey, S.M., Barquet, A.P.B., Kuwabara, L.H., Cauchick Miguel, P.A., Rozenfeld, H., 2016. Business process support for IoT based product-service systems (PSS). Bus. Process Manag. J. 22, 305323. https://doi.org/10.1108/BPMJ-05-2015-0078

Zhang, Y., Li, Y., Sun, Z., Xiong, H., Qin, R., Li, C., 2020. Cost-imbalanced hyper parameter learning framework for quality classification. J. Clean. Prod. 242, 118481. https://doi.org/10.1016/J.JCLEPRO.2019.118481

Zhang, Y., Ren, S., Liu, Y., Sakao, T., Huisingh, D., 2017a. A framework for Big Data driven product lifecycle management. J. Clean. Prod. 159, 229-240. https://doi.org/10.1016/j.jclepro.2017.04.172

Zhang, Y., Ren, S., Liu, Y., Si, S., 2017b. A big data analytics architecture for cleaner manufacturing and maintenance processes of complex products. J. Clean. Prod. 142, 626-641.

https://doi.org/10.1016/j.jclepro.2016.07.123 